Casos Clínicos

Arch. Esp. Urol., 59, 9 (899-901), 2006

\section{NEUROFIBROMA DE LA VEJIGA: CASO CLÍNICO Y REVISIÓN DE LA LITERATURA.}

Pedro Manuel Cabrera Castillo, Sergio Alonso y Gregorio, José Ramón Cansino Alcaide, Alfredo Aguilera Basan y Javier Jesús De La Peña Barthel.

Servicio de Urología. Hospital Universitario La Paz. Madrid. España.

Resumen.- OBJETIVO: La prevalencia de la enfermedad es 1 de cada 3000 nacimientos vivos, siendo más frecuente en hombres que en mujeres con una relación 3: 1 en todas las razas. Los neurofibromas del tracto genitourinario provienen generalmente de los nervios pélvicos, vesicales y del plexo prostático. La vejiga es el órgano más afectado del tracto urinario; la neurofibromatosis vesical puede presentarse como un proceso infiltrativo difuso o como un neurofibroma aislado. En la vejiga los neurofibromas se originan de los ganglios nerviosos de la pared vesical y se tiñen positivamente para la proteína S-100 y el colágeno de tipo IV con técnicas de inmunohistoquímica.

MÉTODOS: Se realiza una revisión de la literatura acerca de neurofibromas en el tracto urinario y en especial del neurofibroma de la vejiga. Presentamos un caso de una mujer de 45 años de edad que acude a la consulta de urología con sindrome miccional e infecciones urinarias de repetición. Se realizan pruebas de imagen en las que se evidencia masa endovesical en cara lateral izquierda de la vejiga y engrosamiento difuso de la pared vesical. El engrosamiento de la vejiga es el hallazgo mas característico en los estudios de imagen que también puede estar presente en otras patologías como son el pseudotumor inflamatorio y el leiomioma, por lo que el diagnóstico final se realiza mediante el estudio anatomo-patólogico

RESULTADOS: Llama la atención en el caso clínico que presentamos, que se trata de una enferma que no tenía diagnóstico previo de neurofibromatosis, siendo la afección vesical la primera manifestación de la enfermedad. Es por eso que en este caso fue difícil sospechar el diagnóstico final dada la ausencia de otras manifestaciones características de la patología. Se realiza resección transuretral del tumor, y el diagnóstico se realiza por el estudio anatomo-patológico e inmunohistoquímico de las muestras obtenidas. Posteriormente se sigue a la enferma en las consultas de urología y es remitida al servicio de Medicina Interna para descartar manifestaciones de la enfermedad en otros órganos

CONCLUSIONES: La neurofibromatosis es una enfermedad sistémica que es muy poco frecuente, y la afectación del tracto urinario por neurofibromas lo es aún más. La vejiga es el órgano que mas frecuentemente se afecta del sistema urinario y suele ser como una infiltración difusa o mas raramente como un tumor solitario. El diagnóstico final es anatomo-patológico e inmunohistoquímico. El tratamiento suele ser conservador. Se debe estudiar al enfermo con el objetivo de descartar manifestaciones de la enfermedad, así como realizar seguimiento para valorar la aparición de nuevas lesiones

Palabras clave: Neurofibromatosis. Vejiga. Estudio inmunohistoquímico

Summary.- OBJECTIVE: The prevalence of the disease is 1/3000 newborns; it is more frequent in men than in women with a 3:1 ratio in all races. Genitourinary tract neurofibromas usually arise from the pelvic and bladder nerves, and the prostatic plexus. Bladder is the most frequently affected organ of the urinary tract; bladder neurofibromatosis may present as a diffuse infiltrative process or an isolated neurofibroma. Bladder neurofibromas arise from nervous ganglia of the bladder wall and stain positive for protein S-100 and type IV collagen with immunohistochemical techniques.

METHODS: We performed a bibliographic review about urinary tract neurofibromas, and specifically of bladder neurofibroma. We report the case of a 45-year-old female consulting for voiding symptoms and recurrent urinary tract infections. Imaging tests showed a mass in the left lateral 
wall of the bladder and diffuse thickening of the bladder wall. The thickening of the bladder wall is the most characteristic finding in imaging tests, which may also be present in other diseases such as inflammatory pseudotumor and leiomyoma, so that final diagnosis should be achieved by pathologic study.

RESULTS: The case is relevant for the absence of previous diagnosis of neurofibromatosis, being bladder involvement its clinical debut. This is why it was difficult to suspect the final diagnosis: the absence of other characteristic clinical manifestations of the disease. Transurethral resection of the tumor was performed and pathologic and immunohistochemical studies offered the final diagnosis. The patient was followed in the urology clinic and also sent to the internal medicine department to rule out other organs involvement of the disease.

CONCLUSIONS: Neurofibromatosis is a rare systemic disease, and urinary tract involvement is rarer. Bladder is the most frequently involved organ in the urinary tract, generally as a diffuse infiltration or more rarely a solitary tumor. The final diagnosis is pathological and immunohistochemical. Treatment is usually conservative. The patient should be worked up to rule out other manifestations of the disease, and followed to evaluate the development of new lesions.

Keywords: Neurofibromatosis. Bladder. Immunohistochemical study.

\section{INTRODUCCIÓN}

La neurofibromatosis es una enfermedad hereditaria autosómica dominante. Se trata de un trastorno que afecta las células de Schwan y los fibroblastos de la vaina de los nervios. Fue descrita por primera vez en 1849 , pero fue realmente von Recklinghausen quien la describió en detalle y en su honor se dió el nombre a la enfermedad. El gen de la neurofibromatosis está ubicado en el cromosoma 17 (1).

La prevalencia de la enfermedad es 1 de cada 3000 nacimientos vivos, siendo más frecuente en hombres que en mujeres con una relación 3:1 en todas las razas (2).

Puede afectar prácticamente cualquier órgano de la anatomía. Los neurofibromas del tracto genitourinario provienen generalmente de los nervios pélvicos, vesicales y del plexo prostático. La vejiga es el órgano más afectado del tracto urinario; la neurofibromatosis vesical puede presentarse como un proceso infiltrativo difuso o como un neurofibroma aislado.
La neurofibromatosis vesical es mas frecuente en niños y en adultos jóvenes y se manifiesta con síntomas de obstrucción del tracto urinario, incontinencia urinaria, irritabilidad vesical, dolor en flanco, hematuria o masa tumoral en la pared vesical (3). Los neurofibromas vesicales pueden identificarse con pruebas de imagen como la ecografía, urografía intravenosa (UIV), Tomografía Axial Computarizada (TAC), cistoscopia y biopsia, que constituyen las pruebas habituales que se realizan para el estudio de afecciones vesicales; el diagnóstico definitivo es por anatomía patológica.

En la vejiga los neurofibromas se originan de los ganglios nerviosos de la pared vesical y se tiñen positivamente para la proteína S-100 y el colágeno de tipo IV con técnicas de inmunohistoquímica.

El tratamiento generalmente es conservador, a menos que se observe dilatación del tracto urinario superior, - la masa produzca pérdida importante del volumen vesical con síntomas discapacitantes. Se debe realizar seguimiento para detectar la posible transformación maligna del tumor hacia un neurofibrosarcoma, lo cual es muy poco frecuente (4).

\section{CASO CLÍNICO}

Mujer de 45 años de edad que acude a la consulta de urología con síndrome miccional e infecciones urinarias de repetición. La paciente tenía antecedentes de hipotiroidismo, hipertensión arterial en tratamiento habitual con atenolol y enalapril, fumadora de 10 cigarrillos día e intervenida de colecistectomía. En la exploración física no se evidenciaron hallazgos patológicos. Se realiza ecografía renal y vesical en la que se objetiva engrosamiento irregular de la pared de la vejiga (Figura 1). Se realiza posteriormente cistoscopia en la cual se evidencia vejiga de poca capacidad con neoformación de aproximadamente 5 centímetros de diámetro de aspecto sólido en la cara lateral izquierda hacia fondo y cara anterior, de aspecto inflamatorio y límites poco definidos. En la TAC abdomino-pélvico (Figura 2), se observa vejiga con engrosamiento difuso de su pared, siendo más importante en el lado izquierdo. Tras la administración de contraste se observa captación difusa de toda la pared vesical con marcado realce de la mucosa parietal izquierda. No se observan adenomegalias mesentéricas ni retroperitoneales. Citología de orina negativa en dos ocasiones para células tumorales.

Ante dichos hallazgos se procede a realizar resección transuretral de neoformación vesical más toma de biopsias vesicales aleatorias.

Las muestras obtenidas fueron enviadas para análisis anatomo-patológico, en el que se evidencia proliferación difusa de células fusiformes y alargadas a nivel de la lámina propia, con gran neoformación vascular; además se observan estructuras nodulares constituídas 


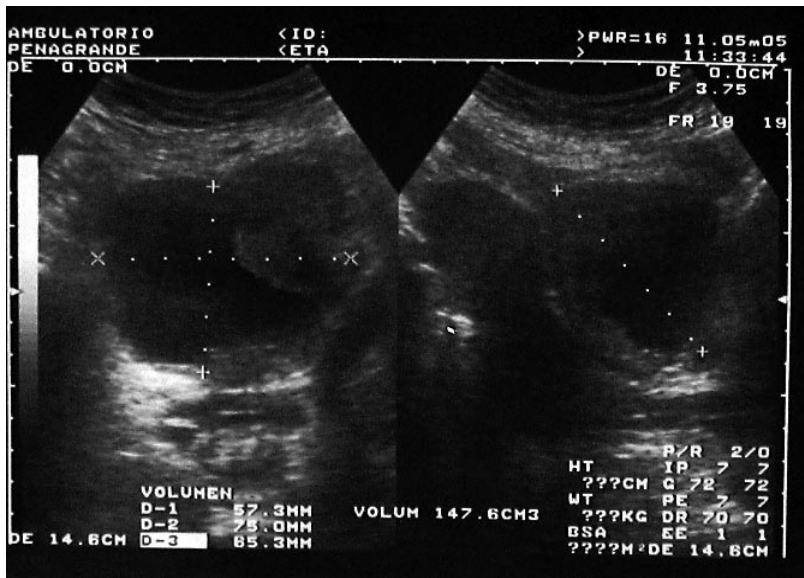

FIGURA 1. Ecografía de vejiga. Se observa el engrosamiento irregular de la pared vesical, más acentuado en la pared lateral izquierda.

por un material eosinófilo acelular cuyas fibras se disponen de forma arremolinada, constituyendo cuerpos de Wagner-Meissner, en disposición concéntrica simulando estructuras nerviosas. Mediante técnicas de inmunohistoquímica se confirma que las células fusiformes y los cuerpos de Wagner-Meissner son intensamente positivos frente al antisuero S-100, por lo cual se confirma el diagnóstico de neurofibromatosis difusa de la pared vesical.

Se remite a la paciente a la consulta de Medicina Interna para continuar con el estudio y descartar otras manifestaciones de neurofibromatosis en otros órganos. De la misma manera la enferma es seguida por la consulta de urología periódicamente para vigilar la aparición de nuevas neoformaciones.

\section{DISCUSIÓN}

La afectación vesical en el contexto de una neurofibromatosis es excepcional, solo existen reportados en la literatura alrededor de 60 casos.

El engrosamiento de la vejiga es el hallazgo mas caraterístico en los estudios de imagen, como se evidenció en la ecografía renal y en la TAC abdomino-pélvica de este caso, aunque también puede presentarse como tumores aislados que protruyen hacia la luz vesical. El engrosamiento de la pared vesical también puede estar presente en otras patologías como son el pseudotumor inflamatorio y el leiomioma, por lo que el diagnóstico final se realiza mediante el estudio anatomo-patólogico, siendo necesario la utilización de técnicas de inmunohistoquímica para diferenciar adecuadamente las células.

Llama la atención en el caso clínico que presentamos, que se trata de una enferma que no tenía diagnóstico previo de neurofibromatosis, siendo la afección vesical

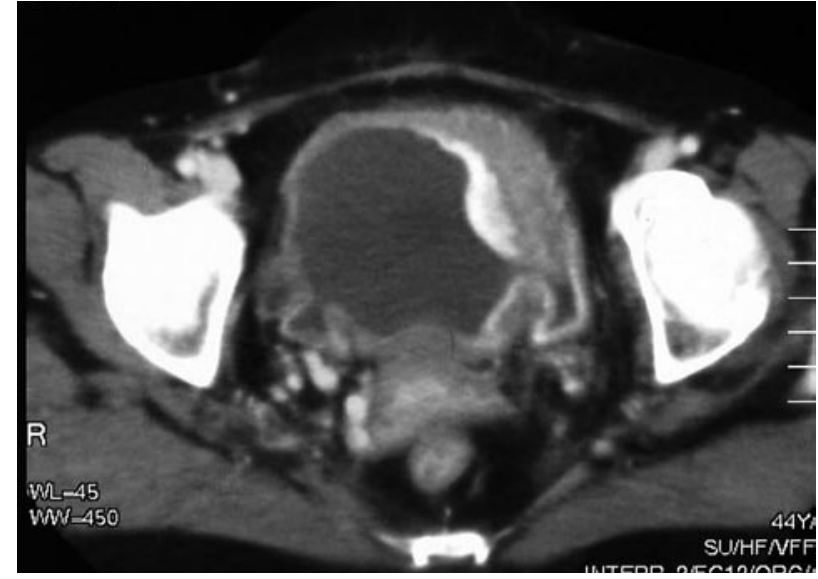

FIGURA 2. Engrosamiento difuso de la pared vesical. Realce tras la administración de contraste, mas acentuado en la pared lateral izquierda.

la primera manifestación de la enfermedad. Es por eso que en este caso fue difícil sospechar el diagnóstico final dada la ausencia de otras manifestaciones características de la patología.

Como se comentó anteriormente, el manejo de la neurofibromatosis vesical suele ser conservador (5), a menos que exista dilatación del tracto urinario superior o pérdida importante del volumen vesical que ocasione síntomas a los enfermos.

Es importante destacar que en estos casos hay que realizar seguimiento períodico de los enfermos ya que el crecimiento del tumor puede ser signo de transformación maligna, lo cual ocurre entre el $5 \%$ y el $10 \%$ de los casos (6); de éstos tumores, el $22 \%$ puede presentar mestástasis (4).

\section{BIBLIOGRAFÍA Y LECTURAS RECOMENDADAS (*lectura de interés $y^{* *}$ lectura fundamental)}

**1. ANIRUDDHA, C.; MALCOM, A.: "Neurofibromatosis involving the urinary bladder". Int. J. Urol., 8: 645, 2001.

2. GOLDBERG, N.S.; ROENIGK, R.K.: "Neurofibromatosis, tuberous sclerosis and other neurocutaneous disorders. Cutaneus Medicine and Surgery. An Integrated Program in Dermatology". WB Saunders, Philadelphia, 1729, 1996.

*3. CHENG, L.; SCHEITHAUER, B.W.: "Neurofibroma of the urinary bladder". Cancer, 86: 505, 1999.

4. CLARK, S.S.; MARLETT, M.M.: "Neurofibromatosis of the bladder in children: Case report and literature review". J. Urol., 118: 654, 1977.

5. INSTA, A.; LINDELL, O.: "Neurofibromatosis of the bladder, case report". Scand. J. Urol. Neprol., 30: 497, 1996.

6. BLUN, M.D.; BAHNSON, R.R.: "Urologic manisfestation of Von Recklinghause's Neurofibromatosis". Urology, 26: 209, 1985. 